\title{
The red giant branch phase transition: Implications for the RGB luminosity function bump and detections of Li-rich red clump stars
}

\author{
Santi Cassisi ${ }^{1,2}$, Maurizio Salaris ${ }^{3}$, and Adriano Pietrinferni ${ }^{1}$ \\ 1 INAF-Osservatorio Astronomico di Teramo, via M. Maggini, 64100 Teramo, Italy \\ e-mail: cassisi@oa-teramo.inaf.it \\ 2 Instituto de Astrofísica de Canarias, Calle via Lactea s/n, 38205 La Laguna, Tenerife, Spain \\ 3 Astrophysics Research Institute, Liverpool John Moores University, IC2, Liverpool Science Park, 146 Brownlow Hill, \\ Liverpool L3 5RF, UK \\ e-mail: M.Salaris@ljmu.ac.uk
}

Received 21 September 2015 / Accepted 1 October 2015

\begin{abstract}
We performed a detailed study of the evolution of the luminosity of He-ignition stage and of the red giant branch bump luminosity during the red giant branch phase transition for various metallicities. To this purpose we calculated a grid of stellar models that sample the mass range of the transition with a fine mass step equal to $0.01 M_{\odot}$. We find that for a stellar population with a given initial chemical composition, there is a critical age (of 1.1-1.2 Gyr) around which a decrease in age of just 20-30 million years causes a drastic drop in the red giant branch tip brightness. We also find a narrow age range (a few $10^{7} \mathrm{yr}$ ) around the transition, characterized by the luminosity of the red giant branch bump being brighter than the luminosity of He ignition. We discuss a possible link between this occurrence and observations of Li-rich core He-burning stars.
\end{abstract}

Key words. stars: evolution - stars: interiors - Hertzsprung-Russell and C-M diagrams - stars: luminosity function, mass function stars: atmospheres

\section{Introduction}

Theoretical stellar evolution calculations show that, for any given chemical composition, there is a minimum initial mass usually named $M_{\mathrm{HeF}}$ - that is able to ignite He-burning at the end of the red giant branch (RGB) phase within a He core not affected by electron degeneracy. Below this threshold, the RGB phase lasts longer and He-burning ignition occurs via a mildly violent He flash in an electron degenerate core. After the detailed analysis by Sweigart et al. (1990), it is customary to use the designation $R G B$ phase transition to describe the changes that occur in both the morphology of the colour-magnitude-diagram and the integrated spectral energy distribution of a stellar population, when stars with mass lower than $M_{\mathrm{HeF}}$ begin to populate the RGB.

All stars with initial masses below $M_{\mathrm{HeF}}$ (that we denote as low-mass stars), owing to the large - and similar - level of electron degeneracy in their cores, reach a similar value of the Hecore mass $\left(M_{\mathrm{cHe}}\right)$ at $\mathrm{He}$ ignition. Owing to the $M_{\mathrm{cHe}}$-luminosity relation for electron degenerate cores, these stars will also attain a similar bolometric luminosity at He ignition, which coincides with the brightest point along the RGB (this latter denoted as RGB tip). When the initial mass reaches values around $M_{\mathrm{HeF}}$, there is a narrow mass range $\left(\sim 0.15 M_{\odot}\right.$; almost independent of chemical composition) populated by objects that do not develop a significant level of electron degeneracy in the He core and that attain He ignition via a very mild He flash. In this regime $M_{\mathrm{cHe}}$ at ignition decreases drastically with increasing stellar mass. Beyond this range, when the initial mass is higher than $M_{\mathrm{HeF}}$, the value of $M_{\mathrm{cHe}}$ at ignition increases almost linearly with mass as a consequence of the increasing mass of the convective core during the previous central H-burning stage.
Given that both luminosity and lifetimes of the following evolutionary phases depend on the value of $M_{\mathrm{cHe}}$ at $\mathrm{He}$ ignition, the discontinuity of the trend of the He-core mass with total mass around $M_{\mathrm{HeF}}$ does affect the properties of core and shell He-burning stages.

At the same time, the differential luminosity function (LF - star counts as a function of magnitude) of the RGB of old stellar populations, such as globular clusters, displays a characteristic local maximum in the star counts, commonly denoted as the RGB bump (Thomas 1967; Iben 1968; King et al. 1985). The luminosity of the RGB bump corresponds to the stage when the $\mathrm{H}$-burning shell crosses the $\mathrm{H}$-abundance discontinuity left over by the outer convection zone at the completion of the first dredge up during the early RGB evolution. During this crossing, the luminosity of the star drops temporarily, before starting to increase again after the $\mathrm{H}$ shell has moved beyond the discontinuity. There is therefore a luminosity range along the RGB that is crossed three times, and this causes the appearance of the bump in the LF. The RGB bump brightness is an important tracer of the interior chemical stratification of low-mass RGB stars, and it has been at the crossroad of several theoretical and observational investigations (see Cassisi \& Salaris 1997; Cassisi et al. 2002; Salaris et al. 2002; Nataf et al. 2013; Nataf 2014; Christensen-Dalsgaard 2015, and references therein).

For a fixed initial stellar mass, the RGB bump becomes brighter when the initial He abundance increases and/or the metallicity decreases, whilst for a given chemical composition, the bump becomes brighter when the initial mass increases. These trends are explained by the varying depth of the convective envelope at the completion of the dredge up. The shallower (in terms of distance from the centre in mass 
units) the convective envelope at the dredge up, the higher the mass of the He-core when the H-burning shell encounters the $\mathrm{H}$-discontinuity, and the brighter the RGB bump. In stars with mass above $M_{\mathrm{HeF}}$, the thermal conditions required for He ignition are achieved earlier, as a consequence of the vanishing level of electron degeneracy, so that triple- $\alpha$ nuclear burning starts before the advancing H-burning shell encounters the H-discontinuity, and the RGB bump disappears.

In this paper we investigate the occurrence of the RGB bump in the thus far unexplored mass range that corresponds to the RGB phase transition. Section 2 describes our models, while Sect. 3 presents the results concerning the RGB bump. Section 4 discusses a possible connection with the presence of lithium-rich red clump stars.

\section{The theoretical models}

All stellar models presented here have been computed with the same code and physics inputs as are employed for the BaSTI ${ }^{1}$ stellar model library (Pietrinferni et al. 2004, 2006), along with a scaled-solar heavy element mixture (see Pietrinferni et al. 2004, for more details). We computed models with convective core overshooting during the main sequence by using the same prescriptions adopted in Pietrinferni et al. (2004), and we mapped the whole RGB phase transition with a very fine mass step of $0.01 M_{\odot}$. This choice allows us to trace any change in the evolutionary properties of stars along the transition, which would probably be missed when adopting a coarser mass grid. Models have been computed for metallicities $Z=0.004(Y=0.251$ mass between 1.75 and $\left.2.00 M_{\odot}\right), Z=0.008(Y=0.256-$ mass between 1.80 and $\left.2.00 M_{\odot}\right)$, and $Z=0.0198(Y=0.2734-$ mass between 1.99 and $2.05 M_{\odot}$ ). These chemical compositions correspond to grid points in the original BaSTI archive, and our calculations effectively represent an extension of the BaSTI model library.

Figure 1 displays selected evolutionary properties at the brightest point along the RGB evolution of our models for $Z=0.004$ and $z=0.008$. The models shown in this figure were computed including convective core overshooting during the central H-burning stage, as in BaSTI calculations. The convective cores have been extended beyond the boundary fixed by the Schwarzschild criterion by an amount $\lambda_{\mathrm{ov}} H_{\mathrm{p}}$, where $H_{\mathrm{p}}$ is the pressure scale height at the Schwarzschild border and $\lambda_{\mathrm{ov}}$ a free parameter. For masses higher than or equal to $1.7 M_{\odot}$, the models employ $\lambda_{\mathrm{ov}}=0.20 H_{\mathrm{p}}$; for stars less massive than $1.1 M_{\odot} \lambda_{\mathrm{ov}}=0$, while in the intermediate range of the model grid $\left(M=1.1,1.2,1.3,1.4,1.5\right.$, and $\left.1.6 M_{\odot}\right), \lambda_{\mathrm{ov}}$ varies as $\lambda_{\mathrm{ov}}=\left(M / M_{\odot}-0.9\right) / 4$ (see Pietrinferni et al. 2004, for details). We did not account for any overshooting from the bottom of the convective envelope.

The RGB transition can be seen at masses around 1.8-1.9 $M_{\odot}$ depending on the metallicity. The core mass $M_{\mathrm{cHe}}$ at He ignition and the RGB tip luminosities drop sharply at $M=1.80 M_{\odot}$ for $Z=0.004$ and $\mathrm{M}=1.89 \mathrm{M}_{\odot}$ for $Z=0.008$, because of the drastic decrease in the level of electron degeneracy in the He core. As shown by panel c of Fig. 1, this drop in the RGB tip luminosity coincides with an unexpected feature. Considering, for example, the $Z=0.004$ models, He ignition occurs at luminosities (also significantly) fainter than the RGB tip for all masses between $\sim 1.79$ and $\sim 1.85 M_{\odot}$. The same behaviour can be seen for the $Z=0.008$ models, although shifted to slightly higher masses, as expected by the increase in the

\footnotetext{
1 http://wWw.oa-teramo.inaf.it/BASTI
}

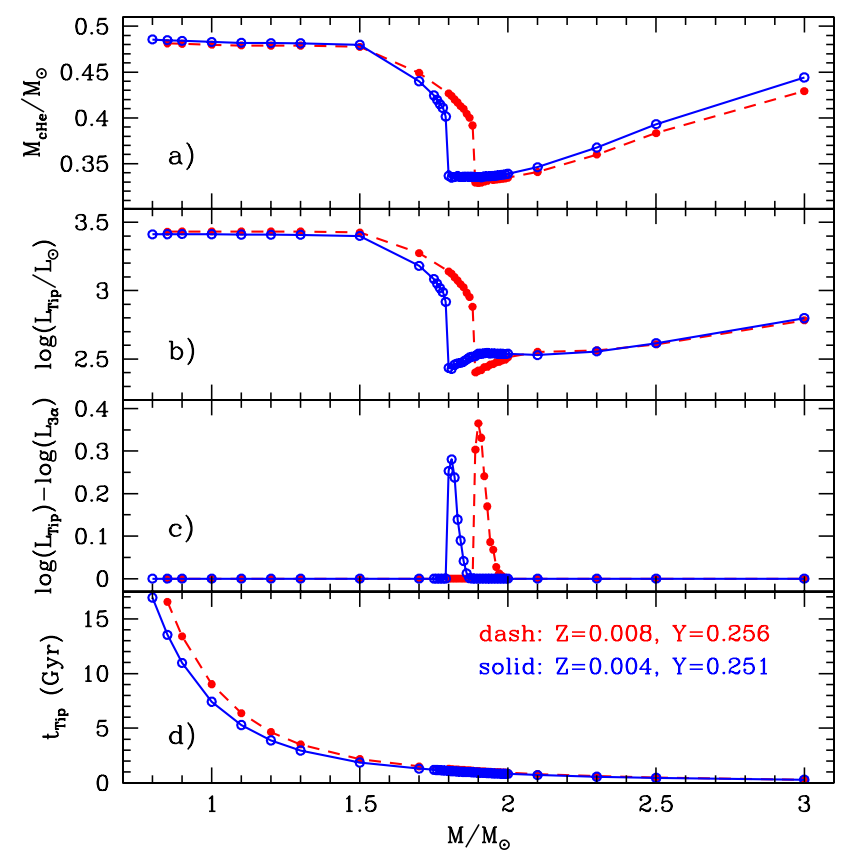

Fig. 1. Selected properties at the RGB tip as a function of the initial total mass for the two labelled chemical compositions: panel a) the He-core mass; panel b) bolometric luminosity; panel c) difference in bolometric luminosity between the RGB tip ( $\left.L_{\mathrm{Tip}}\right)$ and He-ignition; panel d) age.

transition mass $M_{\mathrm{HeF}}$ with metallicity (see Cassisi \& Castellani 1993, and references therein). Outside this narrow mass range, the RGB tip and He ignition do coincide, as usual.

The mass range under scrutiny is so narrow that a very fine mass spacing $\left(\Delta M \sim 0.01 M_{\odot}\right)$ is required to reveal this behaviour. This is why, to the best of our knowledge, it has not been detected in any previous investigation. In the following section, we show how this is related to the combined occurrence of He-burning ignition and the H-burning shell crossing of the $\mathrm{H}$-abundance discontinuity left over at the first dredge up.

\section{The RGB bump luminosity at the RGB phase transition}

Figure 2 shows the evolution of the bolometric luminosity as a function of time during the RGB for models with $Z=0.004$, when sampling the mass range around the minimum of the RGB tip luminosity as shown in Fig. 1. For masses up to $1.79 M_{\odot}$ and with increasing age, the luminosity displays a local maximum and a temporary decrease (the RGB bump) followed by a steep increase up to a maximum value (RGB tip) before a sharp drop after He ignition in the electron degenerate core. This is the standard evolution, with the RGB bump fainter than the tip.

When increasing the mass by only $0.01 M_{\odot}$, the evolution changes drastically. The $1.80 M_{\odot}$ track clearly displays the RGB bump, with the luminosity dropping to a minimum value consistent with the value attained by lower mass models. However, soon after the luminosity starts increasing again (following the standard behaviour) He-burning ignites in the core at a luminosity fainter than the maximum pre-bump value. As a consequence, for this model, which corresponds to the mass with the largest brightness difference between RGB tip and He ignition in panel c of Fig. 1, the RGB tip does not mark the beginning of core He-burning, but instead corresponds to the onset of the RGB bump phase.

The lower panel of Fig. 2 displays the variation with time of both the location (in mass units) of the bottom of the convective 


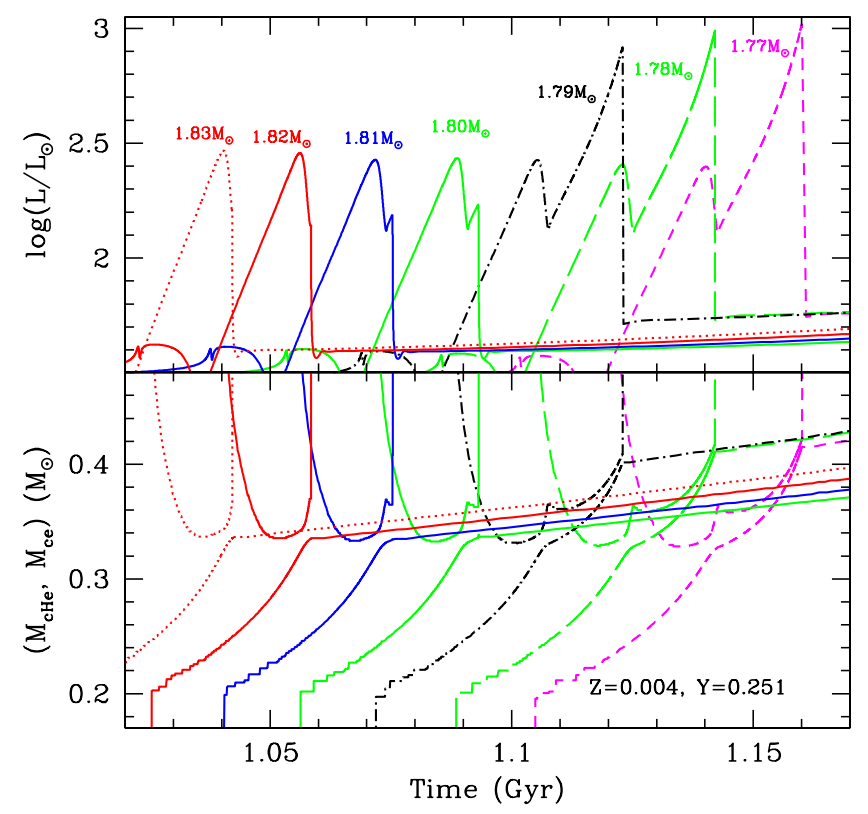

Fig. 2. Top panel: bolometric luminosity as a function of time during the RGB evolution of selected masses (see labels) for $Z=0.004$ and $Y=0.251$. Bottom panel: as in the top panel but for the mass location of the boundary of the $\mathrm{He}$ core and at the bottom of the convective envelope.

envelope and the boundary of the He core, for the same models shown in the top panel. In the $1.80 M_{\odot}$ model, the H-burning shell encounters the $\mathrm{H}$-abundance discontinuity (at the time when the He-core boundary reaches the layer corresponding to the bottom of the convective envelope at its maximum extension) a short time before the triple- $\alpha$ ignition in the core. The signature of He-burning ignition - via an extremely mild He-flash is denoted by the small bump (at $t \sim 1.09 \mathrm{Gyr}$ ) in the trend of the bottom of the convective envelope with time, owing to the energy flux released at He ignition.

The $1.81 M_{\odot}$ displays the same behaviour, but He ignition occurs even earlier, right after the minimum in luminosity of the RGB bump phase. With increasing initial mass, the He ignition occurs progressively earlier, before the luminosity reaches the minimum during the RGB bump phase, and manifests itself as a kink during the decrease in the luminosity due to the crossing of the H-abundance discontinuity. This kink eventually disappears $^{2}$, and for masses greater than $\sim 1.84 M_{\odot}$, He ignition occurs before the $\mathrm{H}$-burning shell encounters the $\mathrm{H}$-abundance discontinuity, so that once again the absolute maximum in luminosity along the RGB marks the start of core He-burning stage.

This behaviour is common to all chemical compositions covered by our analysis. The upper panel of Fig. 3 displays, for example, the trend of the surface luminosity as a function of time during the RGB evolution for models at the RGB transition and initial composition with $Z=0.008$ and $Y=0.256$. The only difference with $Z=0.004$ models is a shift to higher masses, owing to the increase in the transition mass $M_{\mathrm{HeF}}$ with $Z$.

The sharp change in luminosity at He ignition implies that for stellar populations of a given chemical composition there is a critical age equal to $\sim 1.1 \mathrm{Gyr}$ at $Z=0.004$ and $\sim 1.15 \mathrm{Gyr}$ at solar chemical composition, around which a decrease in age of just $2-3 \times 10^{7}$ yr causes a drastic drop in the RGB tip brightness (by $\sim 1.5 \mathrm{mag}$ ). This can be seen clearly in the lower panel of Fig. 3, which displays the $K$-band (that traces well the

\footnotetext{
2 This kink can be barely seen in the $1.82 M_{\odot}$ at $\log \left(L / L_{\odot}\right) \approx 2.142$.
}
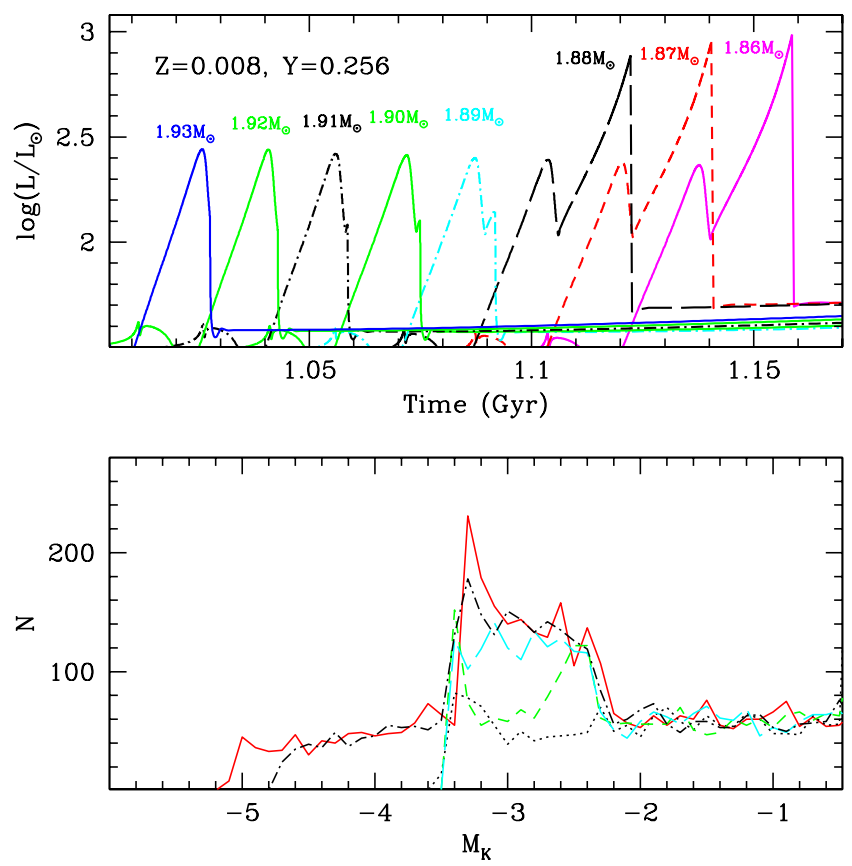

Fig. 3. Top panel: as top panel of fig. 2 but for the chemical composition $Z=0.008, Y=0.256$. Bottom panel: $\mathrm{LF}(0.1 \mathrm{mag}$ bin size $)$ of the upper RGB of $\sim 10^{6} M_{\odot}$ clusters for the same chemical composition, and ages $t=1.14$ (solid line), 1.12 (dotted), 1.10 (short dashed), 1.08 (long dashed), 1.06 (dot-dashed) Gyr, respectively.

bolometric luminosity of RGB stars) LF of $\sim 10^{6} M_{\odot}$ (assuming a Kroupa 2001, initial mass function) synthetic clusters with ages equal to $1.14,1.12,1.10,1.08$, and $1.06 \mathrm{Gyr}(Z=0.008$, $Y-0.256)$, as simulated with our models. The brightness of the RGB tip decreases abruptly by $\sim 1.5$ mag at ages between 1.12 and 1.10 Gyr. At an age of $1.10 \mathrm{Gyr}$ (when the RGB tip coincides with the RGB bump), the number of stars within about one magnitude of the RGB tip is about twice that for the two older ages. This enhanced number of stars near the RGB tip disappears when moving to ages equal to 1.08 and $1.06 \mathrm{Gyr}$, owing to the reduced evolutionary lifetimes of the evolving RGB mass.

The behaviour of the RGB LF around the transition is very difficult to observe. One needs a sample of clusters in the relevant narrow age range discussed here, which is massive enough to have a well-populated upper RGB (we estimate that a total mass of at least $\sim 5 \times 10^{5} M_{\odot}$ is needed) that allow to detect unanbiguously RGB tip and bump, and all approximately at the same distance, or at least with reasonably well determined relative distances.

\section{A link with Li-rich red clump stars?}

Lithium is a fragile element that is easily destroyed by $\alpha$ captures at temperatures higher than $\sim 2.5 \times 10^{6} \mathrm{~K}$. During the early RGB evolution, $\mathrm{Li}$ in the envelope is exposed to the high temperatures of the interiors with a consequent dilution because of the deepening of the outer convection zone. Starting from the present interstellar medium abundance $A(\mathrm{Li}) \sim 3.3 \mathrm{dex}^{3}$ (Grevesse \& Sauval 1998), a post dredge-up $A(\mathrm{Li}) \sim 1.5 \mathrm{dex}$ is expected in Population I stars, the exact value depending on the stellar mass and initial chemical composition (Charbonnel \& Balachandran 2000). Spectroscopic surveys (see, i.e., Brown et al. 1989) have, however, discovered a small fraction $(\sim 1 \%)$

3 As is customary, we define $A(\mathrm{Li})=\log [n(\mathrm{Li}) / n(\mathrm{H})]+12$. 
of RGB stars with Li abundances that exceed this value - hence named $\mathrm{Li}$-rich stars - and indeed they pose a challenge for standard stellar evolution theory.

Several scenarios have been envisaged to explain the occurrence of Li-rich giants (see, i.e., Sackmann \& Boothroyd 1999; Charbonnel \& Balachandran 2000; Denissenkov \& Weiss 2000; Denissenkov 2012, and references therein). For RGB stars brighter than the bump, the general picture (Sackmann \& Boothroyd 1999; Charbonnel \& Balachandran 2000) is that a phase may exist during which newly synthesized Li can be circulated by convection in the outer convective envelope, resulting in a short phase of Li richness. Briefly, the mean molecular weight discontinuity left over by the first dredge-up is expected to strongly inhibit any extra-mixing below the convective envelope. However, when this discontinuity is erased after the RGB bump, extra-mixing processes are possible, as shown by the evolution of the ${ }^{12} \mathrm{C} /{ }^{13} \mathrm{C}$ ratio in field giants at various metallicities (see Charbonnel \& Balachandran 2000, and references therein). The ${ }^{3} \mathrm{He}$ produced through the $p p$-chain on the main sequence and engulfed by the convective envelope during the dredge-up should then first be transported down in the vicinity of the H-burning shell at temperatures high enough for burning through the ${ }^{3} \mathrm{He}(\alpha, \gamma){ }^{7} \mathrm{Be}$ reaction. Then, the produced ${ }^{7} \mathrm{Be}$ should be circulated up to convective layers cool enough where it can decay into ${ }^{7} \mathrm{Li}$ by ${ }^{7} \mathrm{Be}\left(\mathrm{e}^{-}, v\right){ }^{7} \mathrm{Li}$, without ${ }^{7} \mathrm{Li}$ undergoing proton captures. Whether and for how long stars appear Li-rich depends critically on the mass flow rate in the extra-mixing region (Sackmann \& Boothroyd 1999). It is generally expected (e.g., Charbonnel \& Balachandran 2000) that when climbing the RGB beyond the bump, the extra mixing must reach even hotter layers to be able to modify the surface ${ }^{12} \mathrm{C} /{ }^{13} \mathrm{C}$, as observed, with the net effect of Li destruction. Therefore, the RGB Li-rich phase turns out naturally to be a short-lived phase, close to the bump luminosity, as generally observed. (But see Monaco et al. 2011, for observations of Li-rich RGB stars that are much brighter than the bump.)

The discovery of Li-rich RC stars (Kumar et al. 2011; Silva Aguirre et al. 2014) challenges the scenario described above, because RGB stars need to maintain high levels of $\mathrm{Li}$ enrichment from the RGB bump until He ignition. Although it is possible that surface $\mathrm{Li}$ enrichment is a consequence of extramixing processes during the He flash (see Silva Aguirre et al. 2014 , for a discussion on this possibility), it is difficult to reconcile this occurrence with the relative paucity of Li-rich, core He-burning stars. Here we just suggest a working hypothesis to explain at least some RC Li-rich stars, to be further investigated with a detailed analysis.

We have seen that at the RGB phase transition there is a narrow mass range that ignites He shortly after the RGB bump (timescale of the order of $10^{6} \mathrm{yr}$ or less). If extra-mixing processes can efficiently contribute to quickly increasing the surface $\mathrm{Li}$ abundance at the bump, Li production is very likely not followed by any depletion (and change in the ${ }^{12} \mathrm{C} /{ }^{13} \mathrm{C}$ ratio) before He ignition is achieved, so the star should appear as a Li-rich RC object. An important observational consequence is that this class of Li-rich RC stars should show a carbon isotopic ratio similar to the value expected after the first dredgeup. This RGB mass range is extremely narrow, so that the possibility of actually observing the RC progeny of these stars should be extremely small. However, as shown in Fig. 4, models with initial mass around the RGB transition display the longest core He-burning lifetime, as a consequence of the lower He-core mass, hence fainter RC luminosity (see also Cassisi \& Salaris 2013, for a detailed discussion of this issue). This would

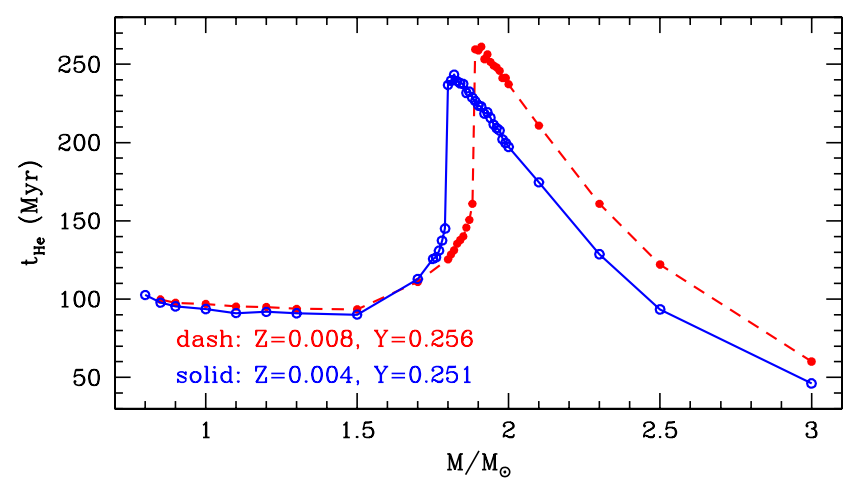

Fig. 4. Lifetime during the central He-burning stage for selected stellar masses along the RGB phase transition, with the labeled chemical compositions.

somewhat increase the probability of observing the RC progeny of RGB transition stars amongst the field disk population.

The RC Li-rich star recently identified by Silva Aguirre et al. (2014) cannot be associated to this potential formation channel, given the lower ${ }^{12} \mathrm{C} /{ }^{13} \mathrm{C}$ compared to the expected post dredgeup value ${ }^{4}$. However, there are Li-rich objects that appear to be $\mathrm{RC}$ stars from their location in the Hertzsprung-Russell diagram and that have a normal post dredge-up ${ }^{12} \mathrm{C} /{ }^{13} \mathrm{C}$ ratio (Kumar et al. 2011). A more precise estimate of their luminosity and $T_{\text {eff }}$ may allow us to better assess both evolutionary status and mass, so as to establish whether they can be related to the progeny of RGB transition objects.

Acknowledgements. S.C. warmly acknowledges financial support by PRININAF2014 (PI: S. Cassisi) and by the Economy and Competitiveness Ministry of the Kingdom of Spain (grant AYA2013-42781-P). A.P. acknowledges financial support by PRIN-INAF2012 (PI: L. Bedin). We thank Laura Greggio for having suggested the calculations that led to these results.

\section{References}

Brown, J. A., Sneden, C., Lambert, D. L., \& Dutchover, Jr., E. 1989, ApJS, 71, 293

Cassisi, S., \& Castellani, V. 1993, ApJS, 88, 509

Cassisi, S., \& Salaris, M. 1997, MNRAS, 285, 593

Cassisi, S., \& Salaris, M. 2013, Old Stellar Populations: How to Study the Fossil Record of Galaxy Formation (Wiley-VCH)

Cassisi, S., Salaris, M., \& Bono, G. 2002, ApJ, 565, 1231

Charbonnel, C., \& Balachandran, S. C. 2000, A\&A, 359, 563

Christensen-Dalsgaard, J. 2015, MNRAS, 453, 666

Denissenkov, P. A. 2012, ApJ, 753, L3

Denissenkov, P. A., \& Weiss, A. 2000, A\&A, 358, L49

Grevesse, N., \& Sauval, A. J. 1998, Space Sci. Rev., 85, 161

Iben, I. 1968, Nature, 220, 143

King, C. R., Da Costa, G. S., \& Demarque, P. 1985, ApJ, 299, 674

Kroupa, P. 2001, MNRAS, 322, 231

Kumar, Y. B., Reddy, B. E., \& Lambert, D. L. 2011, ApJ, 730, L12

Monaco, L., Villanova, S., Moni Bidin, C., et al. 2011, A\&A, 529, A90

Nataf, D. M. 2014, MNRAS, 445, 3839

Nataf, D. M., Gould, A. P., Pinsonneault, M. H., \& Udalski, A. 2013, ApJ, 766, 77

Pietrinferni, A., Cassisi, S., Salaris, M., \& Castelli, F. 2004, ApJ, 612, 168

Pietrinferni, A., Cassisi, S., Salaris, M., \& Castelli, F. 2006, ApJ, 642, 797

Sackmann, I.-J., \& Boothroyd, A. I. 1999, ApJ, 510, 217

Salaris, M., Cassisi, S., \& Weiss, A. 2002, PASP, 114, 375

Silva Aguirre, V., Ruchti, G. R., Hekker, S., et al. 2014, ApJ, 784, L16

Sweigart, A. V., Greggio, L., \& Renzini, A. 1990, ApJ, 364, 527

Thomas, H.-C. 1967, Z. Astrophys., 67, 420 4 Also the estimated mass $M=1.536_{-0.056}^{+0.059} M_{\odot}$ is $\sim 0.4 M_{\odot}$ lower than
the initial mass at the RGB transition for about half-solar metallicity.
However, this could potentially be explained by efficient RGB mass loss. 